\title{
Focal segmental glomerulosclerosis, Coats'-like retinopathy, sensorineural deafness and chromosome 4 duplication: a new association
}

\author{
Ben C. Reynolds • Richard J. L. F. Lemmers • \\ John Tolmie • Allan G. Howatson • David A. Hughes
}

Received: 23 November 2009 /Revised: 11 January 2010 /Accepted: 12 January 2010 /Published online: 27 February 2010

(C) The Author(s) 2010. This article is published with open access at Springerlink.com

\begin{abstract}
We describe the novel association in a girl of nephrotic syndrome due to focal segmental glomerulosclerosis, bilateral sensorineural deafness, basal ganglia calcification, bilateral retinopathy similar to that seen in Coats' disease, with de novo duplication of a subtelomeric region of chromosome $4 \mathrm{q} 35$. The chromosomal duplication was identified during investigation of a possible association with features of fascio-scapulo-humeral dystrophy (FSHD). This duplication has not previously been reported with FSGS and adds to the expanding number of genetic associations with steroid-resistant nephrotic syndrome.
\end{abstract}

Keywords Nephrotic syndrome - Genetic inheritance · Glomerulonephritis

B. C. Reynolds $(\bowtie) \cdot$ D. A. Hughes

Renal Unit, Royal Hospital for Sick Children,

38 Dalnair Street,

Glasgow G3 8SJ, UK

e-mail: pinkdoc@doctors.org.uk

R. J. L. F. Lemmers

Department of Human Genetics,

Leiden University Medical Centre,

Leiden, The Netherlands

J. Tolmie

Department of Clinical Genetics,

Ferguson Smith Centre, Royal Hospital for Sick Children,

Glasgow, UK

\section{A. G. Howatson}

Department of Paediatric Pathology,

Royal Hospital for Sick Children,

Glasgow, UK

\section{Introduction}

We describe a 5-1/2-year-old girl with a prior background of sensorineural deafness, Coat's-like retinopathy, basal ganglia calcification and reflex myoclonus who developed renal impairment secondary to advanced glomerulosclerosis. We identified a duplication of a subtelomeric region of chromosome 4q35 including the D4Z4 repeat array of which contractions are associated with the muscular dystrophy FSHD. We discuss the known genetic implications of D4Z4 and briefly review the genetic basis for nephrotic syndrome. We highlight how D4Z4 duplication could theoretically be associated with nephrotic syndrome, and further discuss the extra-renal manifestations and their associations.

\section{Case report}

A 51/2-year-old girl with a complex medical background was referred with a 12-month history of intermittent peri-orbital and subsequently pretibial oedema. She was hypertensive at $130 / 80 \mathrm{mmHg}$ (95th centile 111/73) with significant microscopic haematuria and heavy proteinuria (protein:creatinine ratio $1.876 \mathrm{~g} / \mathrm{mmol}$ creatinine, normal $<0.020$ ). Plasma biochemistry suggested chronic renal impairment with urea $10.6 \mathrm{mmol} / \mathrm{l}$, creatinine $111 \mu \mathrm{mol} / \mathrm{l}$, calcium $1.76 \mathrm{mmol} / \mathrm{l}$, phosphate $1.99 \mathrm{mmol} / \mathrm{l}$, albumin $12 \mathrm{~g} / \mathrm{l}$, parathormone $25.2 \mathrm{pmol} / \mathrm{l}$. Urinary catecholamines, plasma renin and aldosterone were normal. Anti-neutrophil cytoplasmic antibody (ANCA) and anti-nuclear factor antibody (ANA) were negative. Complement studies showed normal $\mathrm{C} 3$, mildly elevated C4 (0.44 g/l, normal 0.12-0.4 g/l). There was also hypertriglyceridaemia $(21.65 \mathrm{mmol} / \mathrm{l}$, normal $0.4-1.5 \mathrm{mmol} /$ 
1). Renal ultrasound showed mildly echogenic large kidneys (right $9.2 \mathrm{~cm}$, left $9 \mathrm{~cm}$, both just above 95th centile) [1].

Percutaneous renal biopsy demonstrated obsolescent sclerosed glomeruli and glomeruli showing variable degrees of segmental sclerosis, increasing mesangial matrix and capillary loop thickening. No normal glomeruli were present. Hyaline droplets and hyalinosis were visualised in capillary loops. There was tubular atrophy with foci of interstitial chronic inflammatory cell infiltrate. Arterial vessels were normal. Immunofluorescence showed segmental IgM and capillary loop C3 deposition. Electron microscopy, restricted to sclerosed glomeruli demonstrated electron dense material in both capillary loops and mesangium. Appearances were in keeping with advanced focal and segmental glomerulosclerosis (FSGS) of the "not otherwise specified" pattern (Fig. 1).

A 4-week trial of prednisolone $\left(60 \mathrm{mg} / \mathrm{m}^{2} /\right.$ day $)$ for her nephrotic syndrome was unsuccessful in reducing her proteinuria. Angiotensin-converting-enzyme inhibition (ACEI) was introduced in the form of enalapril $(2.5 \mathrm{mg})$ to improve blood pressure control and reduce proteinuria. Nephrotic proteinuria persisted and her function deteriorated so this was removed after several months. Renal function rapidly deteriorated over a course of 7 months so she was commenced on nocturnal automated peritoneal dialysis. Blood pressure is controlled on amlodipine alone. She is currently undergoing transplantation preparation.

The patient originally presented to paediatric services at 8 months of age with left leukocoria and retinal detachment. A pattern of extensive subretinal exudate, dilated tortuous vessels and telangiectasia was consistent with Coats' disease except that the patient was female. She was also identified as having bilateral profound sensorineural deafness. Magnetic resonance imaging (MRI) of the brain was normal. Computed tomography

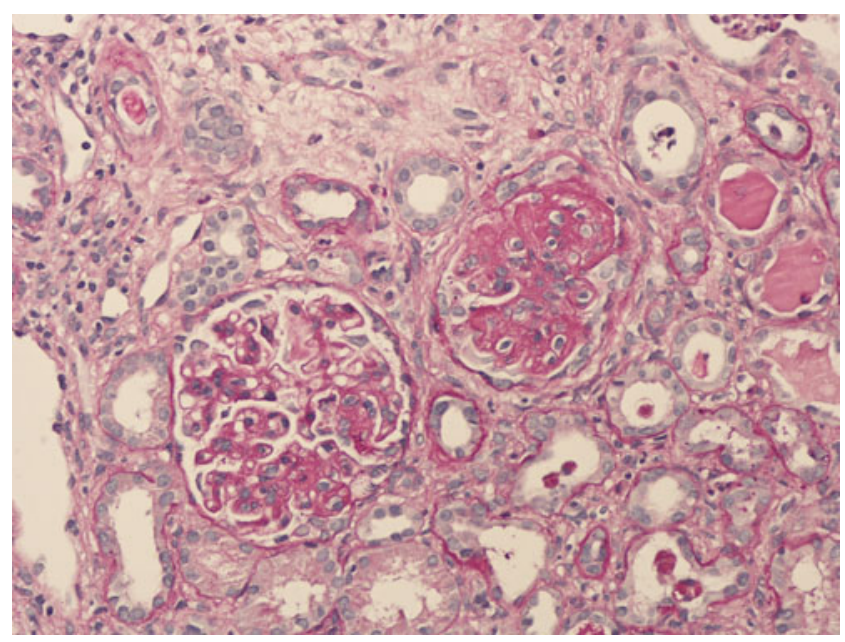

Fig. 1 Haematoxylin and eosin stain demonstrating glomerulosclerosis and mesangial thickening
(CT) assessment for cochlear implantation identified basal ganglia calcification. The cochleae appeared normal.

The patient was also known to the Neurology service with reflex myoclonic jerks triggered by touch only, and three previous tonic-clonic seizures associated with fever. She did not require any anti-epileptic medication. Developmentally, she was identified as delayed, primarily in speech and social functioning, attributed partly to her sensorineural deafness. Cochlear implantation markedly improved this, and she currently attends mainstream education.

The association between Coats'-like retinopathy and sensorineural deafness had been described in fascio-scapulohumeral dystrophy (FSHD). This prompted genetic analysis despite the absence of muscle weakness and a normal muscle biopsy. Initial standard genetic testing for FSHD by linear gel electrophoresis, Southern blotting and hybridisation with probe p13E-11 suggested a de novo contraction of the D4Z4 repeat array at the subtelomere of chromosome 4q, which is well described in FSHD [2]. Subsequently, more detailed genetic analysis of the D4Z4 region was performed by pulsed-field gel electrophoresis and the use of several hybridisation probes (p13E11, D4Z4, A and B) and also by a newly identified simple sequence length polymorphism (SSLP), $3.5 \mathrm{~kb}$ proximal to D4Z4. Hybridisation probes A and $\mathrm{B}$ recognise the region immediately distal to D4Z4 on chromosomes $4 \mathrm{q}$ and 10q. Previously, multiple subtelomeric chromosome $4 \mathrm{q}$ and $10 \mathrm{q}$ variants have been identified, mainly based on the SSLP marker proximal to D4Z4 and probes A and B distal to D4Z4. Interestingly, only one of the 4qA variants, namely 4A161 seems to be permissive to FSHD by D4Z4 contractions, while similar contractions on chromosomes 4qB and 10q do not result in FSHD. Detailed genetic analysis showed that both parents carry one nonpermissive $4 \mathrm{qB}$ chromosome and one permissive 4A161 chromosome, both with a normal sized D4Z4 repeat array. The patient inherited the $4 \mathrm{qB}$ chromosome from her father and the 4A161 chromosome from her mother, but in addition an extra $15-\mathrm{kb}$ fragment is visible upon hybridisation with p13E-11 and D4Z4. This additional short D4Z4 repeat array, did not hybridise to probe A or B, but the SSLP analysis showed that it is derived from the permissive maternal 4A161 chromosome. More extensive Southern blot analysis of NotI-digested DNA fragments confirmed the D4Z4 duplication along with a subtelomeric fragment of at least $100 \mathrm{~kb}$ proximal to D4Z4 [3]. Further investigations, including karyotype and podocin 1 mutation, were normal.

\section{Discussion}

We have described a novel association of Coats'-like retinopathy, sensorineural deafness, basal ganglia calcification, and steroid-resistant nephrotic syndrome with 
histological appearances of FSGS. We have identified duplication of the D4Z4 repeat on chromosome 4q35, which may account for some, or all, of these features.

Contraction of the D4Z4 repeat is associated with FSHD and is often used as a confirmatory genetic test [2]. The D4Z4 array is located in the subtelomeric area of chromosome $4 \mathrm{q}$ and chromosome $10 \mathrm{q}$, and consists of a number of highly conserved repeat units that are $3.3 \mathrm{~kb}$ in size. Normal individuals have 10-100 copies, whereas the presence of 1-10 copies is highly associated with FSHD, though only on a specific haplotype of chromosome $4 \mathrm{qA}$ [2]. Similar D4Z4 repeat contractions on chromosome $4 \mathrm{qB}$ and $10 \mathrm{q}$ are non-pathogenic. Intriguingly, the absence of copies on chromosome 4 (as in monosomy for $4 q$ ) has no FSHD correlate. The D4Z4 repeat unit encodes for a double homeobox protein DUX4, a transcription activator with a role in cellular apoptosis [4]. It has been suggested that stable DUX4 transcription can only emerge from the most distal D4Z4 unit. Recently, it has been shown that several sense and antisense transcripts emerge from FSHD and control myoblast, and therefore the role of these transcripts in FSHD remains unclear.

Various hypotheses exist as to how contraction of the array causes disease, including dysregulation of the normal transcription mechanisms. One such hypothesis involves signalling via CTCF (CCCTC-binding factor) and A-type lamins [5]. CTCF is a highly conserved protein with multiple regulatory roles, including within chromatin modelling [6]. CTCF binding at D4Z4 appears enriched in the FSHD disease model [4], though again the mechanism and effects are uncertain. Much current research focus within nephrotic syndrome centres around the podocyte actin cytoskeleton, and this may provide another cellular target for research. The constellation of symptoms including retinopathy, deafness, nephrosis, and cerebral calcification may also suggest a defect within cilial physiology. The recognition of the pathological importance of ciliopathies is an expanding field [7]. The existence of a genetic duplication in an area highlighted as important in chromatin regulation could be theorised to lead to cilial dysfunction.

In our case, the association among FSHD, retinopathy and sensorineural deafness [8] triggered the genetic analysis, despite the lack of muscle weakness. The more detailed analysis demonstrated duplication rather than contraction of D4Z4. The duplicated short D4Z4 repeat array shows all characteristics of a locus that would otherwise cause FSHD. There are no previous reports of renal disease associated with either FSHD or Coats'-like retinopathy (S. van der Maarel (2010), personal communication). Duplication of D4Z4 has not previously been associated with clinical symptomatology. Duplication within chromosome $4 \mathrm{q} 35$ has been identified in heredi- tary benign intraepithelial dyskeratosis by Allingham et al. [9], but no other associations are reported. No clinical correlation between our patient and that described by Allingham et al. is apparent.

Genetic aetiologies are increasingly recognised in steroid-resistant nephrotic syndrome [10]. Congenital nephrotic syndrome of the Finnish type and mutations in nephrin (NPHS1) have been well characterised. The role of podocin (NPHS2) mutations is well recognised in its association with FSGS-related nephrotic syndrome [11]. Several mutations within NPHS2 have now been consistently identified. Mutation within the WT1 gene is identified in both Frasier and Denys-Drash syndromes. Other genes with established roles in steroid-resistant nephrotic syndrome include $L A M B 2, P L C E 1, A C T N 4$, $C D 2 A P$ and TRPC6 [10]. The increasing recognition of these genes, and the proteins encoded, are elucidating the molecular pathophysiology of FSGS. The advances here have been reviewed recently $[10,12]$.

None of the candidate genes or structural components of the podocyte is known to localise to $4 \mathrm{q} 35$. The pathogenesis of the nephrotic syndrome in our patient is therefore impossible to predict. Unfortunately, the original biopsy demonstrated advanced sclerosed glomeruli preventing definite clarification of the initial histology. This case may highlight another area of interrogation for mutational analysis in children presenting with steroid-resistant FSGS, and offers a further site for investigation in nephrotic syndrome models. It is plausible that characterisation of these gene products may further elucidate the pathological process within nephrotic syndrome.

The extra-renal manifestations also fail to fit a single association. The presence of basal ganglia calcification has been reported with Coats'-like retinopathy in a number of families [13, 14]. Although other features have been recognised in combination, none has included renal disease. The intracranial calcification was identified in our patient, as part of pre-operative assessment for artificial cochlear implantation. It is plausible that there may be other patients with "normal" MR imaging, intracranial calcification and FSGS. Other patients previously described with intracerebral calcification and Coats'-like retinopathy [14] have also been tested for the duplication, but this has not been identified.

Eye abnormalities are associated with $L A M B 2$ mutations in Pierson syndrome [15], though these are distinctly different from our case. Retinal changes are also seen in Tetraspanin-22 mutation, whilst TSPAN7 is associated with developmental delay, and TSPAN24 with glomerulosclerosis and deafness [12]. It could be theorised that the genetic defect in our patient affects multiple transpanin function, leading to this combination of features. Correlation between FSGS and sensorineural deafness is seen in several 
conditions, and may be related to a shared structural anomaly [16]. This correlation has been further highlighted in mutations affecting mitochondrial genes. The multisystemic involvement of seemingly disparate organs could suggest mitochondrial involvement, though the ocular changes in this case are again notably different. Mitochondrial mutations leading to nephrotic syndromes have now been described by several authorities [17]. No prior associations between cortical reflex myoclonus and FSGS have been reported.

\section{Conclusion}

We have described a previously unreported association between duplication of D4Z4 region on chromosome 4q35 and several clinical features, including the development of steroid-resistant nephrotic syndrome with histological appearances of FSGS. This may indicate a further area of interest in the increasing list of genetic factors influencing the development of FSGS.

Acknowledgements Professor Silvere Van der Maarel, Department of Human Genetics, Leiden University Medical Centre, The Netherlands.

Barbara Young, Consultant Pathologist, Western Infirmary Glasgow, UK.

Sameer Zuberi, Consultant Paediatric Neurologist, Royal Hospital for Sick Children, Glasgow, UK.

Open Access This article is distributed under the terms of the Creative Commons Attribution Noncommercial License which permits any noncommercial use, distribution, and reproduction in any medium, provided the original author(s) and source are credited.

\section{References}

1. Keats TE, Sistrom C (2001) Atlas of radiologic measurements, 7th edn. Mosby, St. Louis

2. De Greef JC, Frants RR, van der Maarel SM (2008) Epigenetic mechanisms of facioscapulohumeral muscular dystrophy. Mutat Res 647(1-2):94-102

3. Lemmers RJ, Osborn M, Haaf T, Rogers M, Frants RR, Padberg GW, Cooper DN, van der Maarel SM, Upahyaya M (2003) D4F104S1 deletion in facioscapulohumeral dystrophy: phenotype, size and detection. Neurology 61(2):178-183

4. Snider L, Asawachaicharn A, Tyler AE, Geng LN, Petek LM, Maves L, Miller DG, Lemmers RJ, Winokur ST, Tawil R, van der Maarel SM, Filippova GN, Tapscott SJ (2009) RNA transcripts, miRNA-sized fragments and proteins produced from D4Z4 units: new candidates for the pathophysiology of fascioscapulohumeral dystrophy. Hum Mol Genet 18(13):2414-2430

5. Ottaviani A, Rival-Gervier S, Boussouar A, Foerster A, Rondier D, Sacconi S, Desnuelle C, Gilson E, Magdinier F (2009) The D4Z4 macrosatellite repeat acts as a CTCF and A-type lamins-dependent insulator in fascio-scapular-humeral dystrophy. PLoS Genet 5(2):e1000394

6. Phillips JE, Corces VG (2009) CTCF: master weaver of the genome. Cell 137(7):1194-1211

7. Baker K, Beales PL (2009) Making sense of cilia in disease: the human ciliopathies. Am J Med Genet Part C Semin Med Genet 151C(4):281-295

8. Gierson MA, Korthals JK, Kousseff BG, Opitz JM, Reynolds JF (2005) Facioscapulohumeral dystrophy with cochlear hearing loss and tortuosity of retinal vessels. Am J Med Gen 22(1):143-147

9. Allingham RR, Seo B, Rampersaud E, Bembe M, Challa P, Liu N, Parrish T, Karolak L, Gilbert J, Pericak-Vance MA, Klintworth GK, Vance JM (2001) A duplication in chromosome 4q35 is associated with hereditary benign intraepithelial dyskeratosis. Am J Hum Genet 68(2):491-494

10. Pollak M (2002) Inherited podocytopathies: FSGS and nephrotic syndrome from a genetic viewpoint. J Am Soc Nephrol 13 (12):3016-3023

11. Hinkes B, Vlangos C, Heeringa S, Mucha B, Gbadegesin R, Liu J, Hasselbacher K, Ozaltin F, Hildebrandt F, APN Study Group (2008) Specific podocin mutations correlate with age of onset in steroid-resistant nephrotic syndrome. J Am Soc Nephrol 19 (2):365-371

12. D'Agati VD (2008) The spectrum of focal segmental glomerulosclerosis: new insights. Curr Opin Nephrol Hypertens 17 (3):271-281

13. Goutieres F, Dollfus H, Becquet F, Dufier JL (1999) Extensive brain calcification in two children with bilateral Coats' disease. Neuropediatrics 30(1):19-21

14. Crow YJ, McMenamin J, Haenggeli CA, Hadley DM, Tirupathi S, Treacy EP, Zuberi SM, Browne BH, Tolmie JL, Stephenson JB (2004) Coats' plus: a progressive familial syndrome of bilateral Coats' disease, characteristic cerebral calcification, leukoencepehalopathy, slow pre- and post-natal linear growth and defects of bone marrow and integument. Neuropediatrics 35(1):10-19

15. Zenker M, Aigner T, Wendler O, Tralau T, Muntefering H, Fenski R, Pitz S, Schumacher V, Royer-Pokora B, Wuhl E, Cochat P, Bouvier R, Kraus C, Mark K, Madlon H, Dotsch J, Rascher W, Maruniak-Chudek I, Lennert T, Neumann LM, Reis A (2004) Human laminin B2 deficiency causes congenital nephrosis with mesangial sclerosis and distinct eye abnormalities. Hum Mol Genet 13(21):2625-2632

16. Prakash S, Chung KW, Sinha S, Barmada M, Ellis D, Ferrell RE, Finegold DN, Randhawa PS, Dinda A, Vats A (2003) Autosomal dominant progressive nephropathy with deafness: linkage to a new locus on chromosome 11q24. J Am Soc Nephrol 14(7):1794-1803

17. Lowik MM, Groenen PJ, Levtchenko EN, Monnens LA, van den Heuvel LP (2009) Molecular genetic analysis of podocyte genes in focal segmental glomerulosclerosis - a review. Eur J Pediatr 168(11):1291-1304 